\title{
Power Factor Correction with Synchronous Condenser for Power Quality Improvement in Industrial Load
}

\author{
Moe Kay Thi Khaing \\ Mandalay Technological University \\ Mandalay, Myanmar
}

\begin{abstract}
As most of loads are of inductive type, the power system is in lagging power factor and as a result poor power quality is occurred. And it is demanded to make power factor correction to be better power quality of power system. Among power factor correction devices, synchronous condenser is studied in this paper to improve the industrial loads. There are ten numbers of induction motors in Tharpaung pulp plant and the net power factor of the factory power system is round about 0.85 lagging. By using 0.75 leading power factor of synchronous condenser, the system power factor is improved to 0.969 lagging. And it is studied to choose the appropriated synchronous condenser of power factor and rating for various system power factors. And if there are different operating times for each induction motors, which synchronous condenser should be run is studied in detail in the paper. It is shown that the use of synchronous condenser improves the system power factor and makes power quality better. In this study, MATLAB program is used for overall plant power factor calculation.
\end{abstract}

Keywords: inductive type, power factor, power quality, synchronous condenser, induction motor, lagging, leading

\section{INTRODUCTION}

Power quality is an important issue for distribution network companies. They must guarantee the electricity supply for the customers, while fulfilling certain quality requirements. The power quality problems with end-users in industrial load are power factor, under voltage, over voltage, voltage sag, voltage swell, voltage fluctuation, grounding, etc. Power factor is also a power quality issue in that low power factor can sometimes cause equipment to fail. In many instances, the cost of low power factor can be high; utilities penalize facilities that have low power factor because they find it difficult to meet the resulting demands for electrical energy. The study of power quality is about optimizing the performance of the power system at the lowest possible operating cost. Power factor is definitely an issue that qualifies on both counts [1].

Synchronous condensers are used principally in large power applications because of their high operating efficiency, reliability, controllable power factor, and relatively low sensitivity to voltage dips. They are constant-speed machines with applications in mills, refineries, power plants, to drive pumps, compressors, fans, pulverizers, and other large loads, and to assist in power factor correction. They are designed specifically for power factor control and have no external shafts, and are called synchronous condensers. It is floating on the system. It behaves as a variable capacitor when the field is over-excited, and as a variable inductor when the field is underexcited. In general, over excitation will cause the synchronous motor to operate at a leading power factor, while under exercitation will cause the motor to operate at a lagging power factor. Synchronous motor can also be used for power-factor correction in an industrial plant consisting of a large number of induction motors.

Power factor can be corrected with a capacitor. However, if a synchronous condenser is run with a leading power factor, it can perform useful work and correct power factor at the same time. In industrial applications where both synchronous condensers and induction motors are used, the synchronous condenser is usually operated at a leading power factor to compensate for the lagging power of the induction motors. An improved power also reduces the system voltage drop and the voltage drop at the terminals. Due to these advantages, synchronous condensers are used to improve power factor of the electrical system of a plant.
Therefore, appropriate rating of synchronous condenser is used with induction motors plant in this paper.

\section{POWER FACTOR CORRECTION BY USING SYNCHRONOUS CONDENSER}

Power factor correction usually means the practice of generating reactive power as close as possible to the load which requires it, rather than supplying it from a remote power station. The objective of power factor correction is to reduce the current flowing in the circuit connecting the load with the source of supply. When the power factor is high, the reactive component is a small percentage of the total and so a given change in the reactive component produces only a small change in the total. Accordingly, it is unnecessary to correct the power factor to exactly unity.

The power factor of a system can be improved by using a synchronous condenser and by varying its excitation. Increasing the strength of the magnets will increase the magnetic attraction and thereby the rotor magnets to have a closer alignment with the corresponding opposite poles of the rotating stator flux, the result is a smaller power angle. Assuming a constant shaft load, the steady-state value of $E_{f} \sin \delta$ must be constant. A step increase in $\mathrm{E}_{\mathrm{f}}$ will cause a transient increase in $\mathrm{E}_{\mathrm{f}} \sin \delta$, and the rotor will accelerate. As the rotor changes its angular position, $\delta$ decreases until $\mathrm{E}_{\mathrm{f}} \sin \delta$ has the same steady-state value as before, at which time the rotor is again operating at synchronous speed. The change in angular position of the rotor magnets relative to the rotating flux of the stator occurs in a fraction of a second [2]. The effect of changes in field excitation on armature current, power angle, and power factor of a synchronous motor operating with a constant shaft load, from a constant voltage, constant frequency supply, is illustrated in Figure 1. For a constant shaft load, $\mathrm{E}_{\mathrm{f} 1} \sin \delta_{1}=\mathrm{E}_{\mathrm{f} 2} \sin \delta_{2}=\mathrm{E}_{\mathrm{f} 3} \sin \delta_{3}=\mathrm{E}_{\mathrm{f}} \sin \delta$ $\mathrm{I}_{\mathrm{a} 1} \cos \Phi_{\mathrm{i} 1}=\mathrm{I}_{\mathrm{a} 2} \cos \Phi_{2}=\mathrm{I}_{\mathrm{a} 3} \cos \Phi_{3}=\mathrm{I}_{\mathrm{a}} \cos \Phi_{\mathrm{i}}$

This is shown in Figure 1, where the locus of the tip of the $I_{a}$ phasor is a line perpendicular to the $\mathrm{V}_{\mathrm{T}}$ phasor. Increasing the excitation from $E_{f 1}$ to $E_{f 3}$ in Figure 1 caused the angle of the current phasor to go from lagging to leading. The value of field excitation that results in unity power factor is called normal excitation. Excitation greater than normal is called overexcitation, and excitation less than normal is called 
underexcitation. Furthermore, as indicated in Figure 1, when operating in the overexcited mode, $\left|E_{\mathrm{f}}\right|>\left|\mathrm{V}_{\mathrm{T}}\right|$.

At minimum stator current, the motor is operating at unity power. If the field current value is held constant, Figure 2 will show the amount of leading kVA supplied to the line. At full load, the field excitation is sufficient to operate the motor at unity power factor for supplying no kVAR. The size of generator, transmission lines, transformers and distribution networks are based on the kVA demand, and the overall losses in these components are also determined by kVA. The electrical power in kilowatts consumed largely determines the fuel consume by the prime mover but in most systems the charges for electrical energy based on kVA demand and on kilowatts. It is usually economic, therefore, to operate a load of some power factor approaching unity, and when the power factor of a load is slow, the saving in power charges obtained by improving the power factor justifies the installation of special equipment for the purposes. The main advantage possessed by synchronous condenser is that the kVAR taken by it can be varied by adjusting the excitation. When such a machine is used to provide the power factor of a load, automatic control excitation can be used to keep the overall load power factor constant, within the capacity of the condenser by using a scheme such as has been described for a synchronous motor. In many cases, operation with fixed excitation is satisfactory and the condenser is then arranged to compensate for a fixed amount of lagging current taken by the load. For each load, there is definite limit beyond which it is not economical to raise the power factor as the increase in cost of equipment required is not compensated for by the reduction in power charges. Figure 3 shows the variation of percentage reduction in $\mathrm{kVA}$ demand for a fixed kilowatt load with the amount of power factor correction expressed as a percentage of the original $\mathrm{kVA}$ curves are shown for several values of power factor of the original load. The reduction in kVA demand can be increased until the maximum value, at unity power factor, is reached. The saving in power charges can be elevated for various size of condenser and the economic limit thus determined[3].

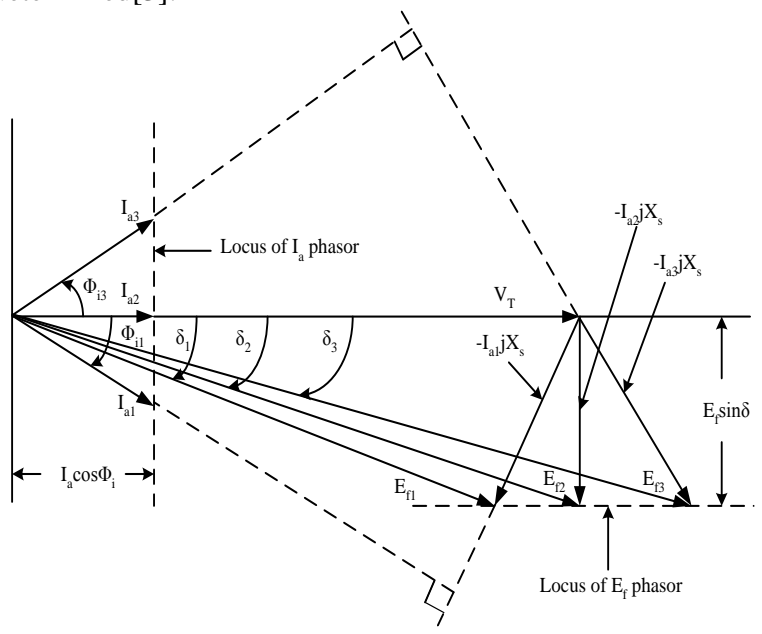

Figure. 1 Phasor diagram showing the effect of changes in field excitation on armature current, power angle, and power factor of a synchronous motor

The maximum amount of reduction in kVA which can be obtained increases of the original factor decreases, but it also involved as increase in the amount of condenser capacity required. The rate of change of reduction in $\mathrm{kVA}$ with increase in condenser rating decreases from the initial value to zero and it is therefore possible, by substituting appropriate saving in cost due to reduced $\mathrm{kVAR}$ and capital cost required for condenser capacity to determine the most economic size of condenser for a given load condition.

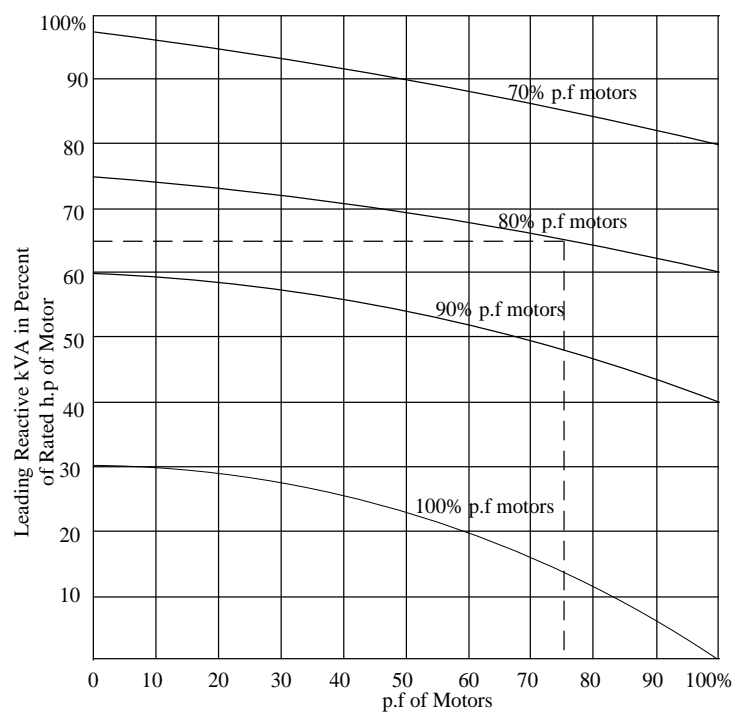

Figure. 2 Showing approximate leading kVA drawn by high speed synchronous motors operate at partial loads with full-load excitation maintained[4]

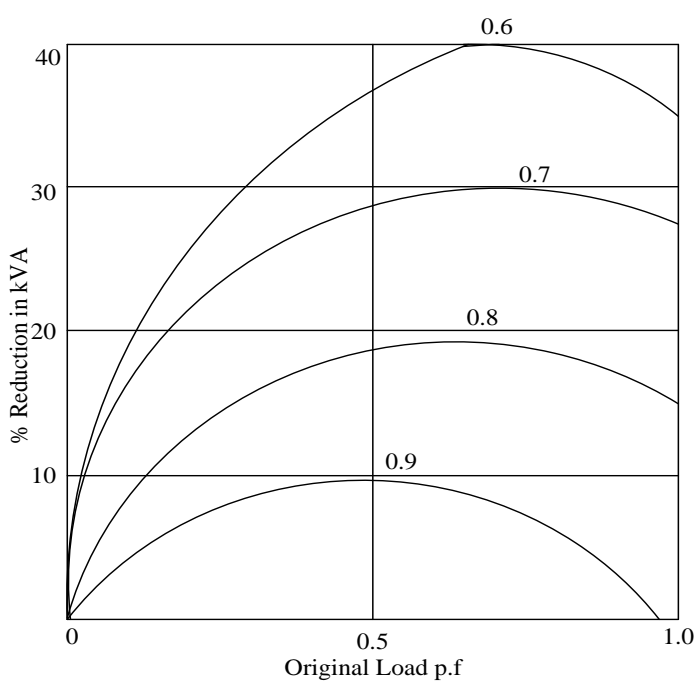

Figure. 3 Relationship between condenser rating reductions in kVA demand [3]

The real input power of the motor $(\mathrm{kW})$ is:

$$
\mathrm{P}=\frac{\text { Output }}{\text { effeciency }}
$$
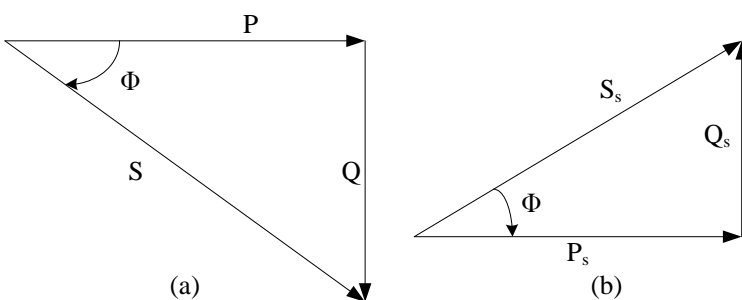

(b) 
Figure. 4 Phasor diagram of (a) induction motor and

$$
\text { (b) synchronous motor }
$$

The power factor (p.f) is:

$$
\text { p.f }=\cos \Phi=\frac{P}{S}
$$

The reactive power( $\mathrm{kVAR})$ is:

$\mathrm{Q}=\mathrm{P} \tan \Phi$

If all loads are induction motors in the system, total real power and reactive power of the system are:

$$
\begin{gathered}
\mathrm{P}_{\mathrm{T}}=\sum_{\mathrm{i}=1}^{\mathrm{n}} \mathrm{Pi}_{\mathrm{i}} \\
\mathrm{Q}_{\mathrm{T}}=\sum_{\mathrm{i}=1}^{\mathrm{n}} \mathrm{Q}_{\mathrm{i}}
\end{gathered}
$$

If a synchronous condenser is added in the system of induction motors, total real power and reactive power are:

$$
\begin{aligned}
& \mathrm{P}_{\mathrm{T}}=\sum_{\mathrm{i}=1}^{\mathrm{n}} \mathrm{Pi}_{\mathrm{i}}+\mathrm{P}_{\mathrm{S}} \\
& \mathrm{Q}_{\mathrm{T}}=\sum_{\mathrm{i}=1}^{\mathrm{n}} \mathrm{Q}_{\mathrm{i}}-\mathrm{Q}_{\mathrm{S}}
\end{aligned}
$$

Power factor of the system is:

$$
\text { p. } \mathrm{f}_{\mathrm{T}}=\cos \left(\tan ^{-1}\left(\frac{\mathrm{QT}}{\mathrm{PT}}\right)\right)
$$

If Total reactive power of induction motors is greater than reactive power of synchronous condenser, power factor of the system is lagging. Otherwise, it is leading.

Where, $\mathrm{S}=$ apparent power(kVA)

$\mathrm{P}_{\mathrm{S}}=$ real power of synchronous condenser

$\mathrm{Q}_{\mathrm{S}}=$ reactive power of synchronous condenser

\section{SYSTEM OF CASE STUDY}

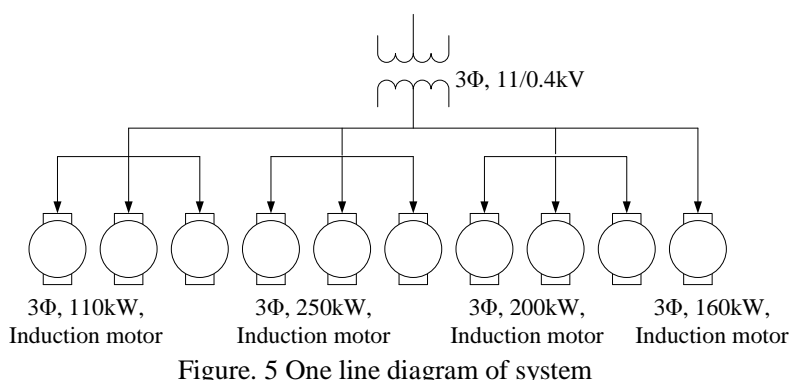

The designed paper plant consists of ten numbers of induction motors for paper making. There are three numbers of $110 \mathrm{~kW}$ induction motor used for first refiner, three numbers of 250 $\mathrm{kW}$ induction motor used for second refiner, three numbers of $200 \mathrm{~kW}$ induction motor used for third refiner and a $160 \mathrm{~kW}$ induction motor used for Hydrapulper. The desired plant is shown in Figure 5. These machines are operated with full load or light load. And then they are operated with all together or some of them. Therefore, various cases are considered in this study.

Table 1. Total installed ratings

\begin{tabular}{|l|l|l|l|l|l|}
\hline Output & $\boldsymbol{\eta}$ & p.f & No: & \multicolumn{2}{|l|}{ Total absorption } \\
\cline { 5 - 6 }$(\mathbf{k W})$ & \multirow{2}{*}{$\boldsymbol{\%})$} & & & $\mathbf{P}(\mathbf{k W})$ & Q(kVAR) \\
\hline 110 & 94 & 0.86 & 3 & 351.064 & 208.306 \\
\hline
\end{tabular}

\begin{tabular}{|l|l|l|l|l|l|}
\hline 250 & 94.5 & 0.81 & 3 & 793.651 & 574.592 \\
\hline 200 & 94.6 & 0.88 & 3 & 634.249 & 342.337 \\
\hline 160 & 94.2 & 0.86 & 1 & 169.851 & 100.782 \\
\hline \multicolumn{5}{|l}{ Total } \\
\hline \multicolumn{2}{|l|}{ p.f of the system } & 1948.815 & 1226.017 \\
\hline
\end{tabular}

\section{COMPARISON RESULTS BY USING DIFFERENT RATINGS OF SYNCHRONOUS CONDENSER}

There are different ratings of synchronous motors available in the market. The study is done based on the rating of $210 \mathrm{~kW}$, $480 \mathrm{~kW}, 600 \mathrm{~kW}, 800 \mathrm{~kW}, 1400 \mathrm{~kW}$ and $2400 \mathrm{~kW}$. Different rating of synchronous motor varies the overall plant power factor. The power factor of overall plant is limited within 0.95 lagging and unity. Various ratings of synchronous motor are

\begin{tabular}{|c|c|c|c|c|c|c|}
\hline \multirow{2}{*}{$\begin{array}{l}\% \\
\text { of } \\
\text { load }\end{array}$} & \multicolumn{6}{|c|}{ p.f of synchronous condenser } \\
\hline & $\begin{array}{l}0.75 \\
\text { (lead:) }\end{array}$ & $\begin{array}{l}0.8 \\
\text { (lead:) }\end{array}$ & $\begin{array}{l}0.85 \\
\text { (lead:) }\end{array}$ & $\begin{array}{l}0.9 \\
\text { (lead:) }\end{array}$ & $\begin{array}{l}0.95 \\
\text { (lead:) }\end{array}$ & 1 \\
\hline 50 & $\begin{array}{l}0.9941 \\
\text { (lag:) }\end{array}$ & $\begin{array}{l}0.9882 \\
\text { (lag:) }\end{array}$ & $\begin{array}{l}0.9806 \\
\text { (lag:) }\end{array}$ & $\begin{array}{l}0.9710 \\
\text { (lag:) }\end{array}$ & $\begin{array}{l}0.9579 \\
\text { (lag:) }\end{array}$ & $\begin{array}{l}0.9243 \\
\text { (lag:) }\end{array}$ \\
\hline 75 & $\begin{array}{l}0.9729 \\
\text { (lag:) }\end{array}$ & $\begin{array}{l}0.9649 \\
\text { (lag:) }\end{array}$ & $\begin{array}{l}0.9563 \\
\text { (lag:) }\end{array}$ & $\begin{array}{l}0.9465 \\
\text { (lag:) }\end{array}$ & $\begin{array}{l}0.9344 \\
\text { (lag:) }\end{array}$ & $\begin{array}{l}0.9063 \\
\text { (lag:) }\end{array}$ \\
\hline 100 & $\begin{array}{l}0.9537 \\
\text { (lag:) }\end{array}$ & $\begin{array}{l}0.9460 \\
\text { (lag:) }\end{array}$ & $\begin{array}{l}0.9379 \\
\text { (lag:) }\end{array}$ & $\begin{array}{l}0.9291 \\
\text { (lag:) }\end{array}$ & $\begin{array}{l}0.9186 \\
\text { (lag:) }\end{array}$ & $\begin{array}{l}0.8950 \\
\text { (lag:) }\end{array}$ \\
\hline 125 & $\begin{array}{l}0.9387 \\
\text { (lag:) }\end{array}$ & $\begin{array}{l}0.9316 \\
\text { (lag:) }\end{array}$ & $\begin{array}{l}0.9244 \\
\text { (lag:) }\end{array}$ & $\begin{array}{l}0.9167 \\
\text { (lag:) }\end{array}$ & $\begin{array}{l}0.9074 \\
\text { (lag:) }\end{array}$ & $\begin{array}{l}0.8872 \\
\text { (lag:) }\end{array}$ \\
\hline
\end{tabular}
considered at 0.75 leading, 0.8 leading, 0.85 leading, 0.9 leading, 0.95 leading and unity of power factor for $50 \%, 75 \%$, $100 \%$ and $125 \%$ change of load.

Table 2. Results by using $210 \mathrm{~kW}$ synchronous condenser

\begin{tabular}{|l|l|l|l|l|l|l|}
\hline \multirow{2}{*}{$\begin{array}{l}\text { \% } \\
\text { of } \\
\text { load }\end{array}$} & $\begin{array}{l}\mathbf{0 . 7 5} \\
\text { (lead:) }\end{array}$ & $\begin{array}{l}\mathbf{0 . 8} \\
\text { (lead:) }\end{array}$ & $\begin{array}{l}\mathbf{0 . 8 5} \\
\text { (lead:) }\end{array}$ & $\begin{array}{l}\mathbf{0 . 9} \\
\text { (lead:) }\end{array}$ & $\begin{array}{l}\mathbf{0 . 9 5} \\
\text { (lead:) }\end{array}$ & $\mathbf{1}$ \\
\hline \multirow{2}{*}{50} & $\begin{array}{l}0.9447 \\
\text { (lag:) }\end{array}$ & $\begin{array}{l}0.9373 \\
\text { (lag:) }\end{array}$ & $\begin{array}{l}0.9297 \\
\text { (lag:) }\end{array}$ & $\begin{array}{l}0.9215 \\
\text { (lag:) }\end{array}$ & $\begin{array}{l}0.9118 \\
\text { (lag:) }\end{array}$ & $\begin{array}{l}0.8902 \\
\text { (lag:) }\end{array}$ \\
\hline 75 & $\begin{array}{l}0.9191 \\
\text { (lag:) }\end{array}$ & $\begin{array}{l}0.9132 \\
\text { (lag:) }\end{array}$ & $\begin{array}{l}0.9073 \\
\text { (lag:) }\end{array}$ & $\begin{array}{l}0.901 \\
\text { (lag:) }\end{array}$ & $\begin{array}{l}0.8937 \\
\text { (lag:) }\end{array}$ & $\begin{array}{l}0.8778 \\
\text { (lag:) }\end{array}$ \\
\hline 100 & $\begin{array}{l}0.9040 \\
\text { (lag:) }\end{array}$ & $\begin{array}{l}0.8989 \\
\text { (lag:) }\end{array}$ & $\begin{array}{l}0.8942 \\
\text { (lag:) }\end{array}$ & $\begin{array}{l}0.8892 \\
\text { (lag:) }\end{array}$ & $\begin{array}{l}0.8834 \\
\text { (lag:) }\end{array}$ & $\begin{array}{l}0.8709 \\
\text { (lag:) }\end{array}$ \\
\hline 125 & $\begin{array}{ll}\text { lag } \\
\text { (lag:) }\end{array}$ & $\begin{array}{l}0.8897 \\
\text { (lag:) }\end{array}$ & $\begin{array}{l}0.8857 \\
\text { (lag:) }\end{array}$ & $\begin{array}{l}0.8815 \\
\text { (lag:) }\end{array}$ & $\begin{array}{l}0.8767 \\
\text { (lag:) }\end{array}$ & $\begin{array}{l}0.8664 \\
\text { (lag:) }\end{array}$ \\
\hline
\end{tabular}

Table 3. Results by using $480 \mathrm{~kW}$ synchronous condenser 
International Journal of Science and Engineering Applications

Volume 3 Issue 3, 2014, ISSN-2319-7560 (Online)

Table 4. Result by using $600 \mathrm{~kW}$ synchronous motor

\begin{tabular}{|l|c|c|c|c|c|c|}
\hline \multirow{2}{*}{\begin{tabular}{l}
\multirow{2}{*}{$\begin{array}{l}\text { of } \\
\text { load }\end{array}$} \\
\cline { 2 - 7 }
\end{tabular}} & $\begin{array}{c}\mathbf{0 . 7 5} \\
\text { (lead:) }\end{array}$ & $\begin{array}{c}\mathbf{0 . 8} \\
\text { (lead:) }\end{array}$ & $\begin{array}{c}\mathbf{0 . 8 5} \\
\text { (lead:) }\end{array}$ & $\begin{array}{l}\mathbf{0 . 9} \\
\text { (lead:) }\end{array}$ & $\begin{array}{c}\mathbf{0 . 9 5} \\
\text { (lead:) }\end{array}$ & $\mathbf{1}$ \\
\hline 50 & $\begin{array}{c}0.9995 \\
\text { (lag:) }\end{array}$ & $\begin{array}{c}0.9966 \\
\text { (lag:) }\end{array}$ & $\begin{array}{c}0.9910 \\
\text { (lag:) }\end{array}$ & $\begin{array}{c}0.9827 \\
\text { (lag:) }\end{array}$ & $\begin{array}{c}0.9701 \\
\text { (lag:) }\end{array}$ & $\begin{array}{c}0.9347 \\
\text { (lag:) }\end{array}$ \\
\hline 75 & $\begin{array}{c}0.9859 \\
\text { (lag:) }\end{array}$ & $\begin{array}{c}0.9787 \\
\text { (lag:) }\end{array}$ & $\begin{array}{c}0.9703 \\
\text { (lag:) }\end{array}$ & $\begin{array}{c}0.9603 \\
\text { (lag:) }\end{array}$ & $\begin{array}{c}0.9474 \\
\text { (lag:) }\end{array}$ & $\begin{array}{c}0.9160 \\
\text { (lag:) }\end{array}$ \\
\hline \multirow{2}{*}{100} & $\begin{array}{c}0.9687 \\
\text { (lag:) }\end{array}$ & $\begin{array}{c}0.9607 \\
\text { (lag:) }\end{array}$ & $\begin{array}{c}0.9522 \\
\text { (lag:) }\end{array}$ & $\begin{array}{c}0.9426 \\
\text { (lag:) }\end{array}$ & $\begin{array}{c}0.9307 \\
\text { (lag:) }\end{array}$ & $\begin{array}{c}0.9037 \\
\text { (lag:) }\end{array}$ \\
\hline \multirow{2}{*}{125} & $\begin{array}{c}0.9537 \\
\text { (lag:) }\end{array}$ & $\begin{array}{c}0.9460 \\
\text { (lag:) }\end{array}$ & $\begin{array}{c}0.9379 \\
\text { (lag:) }\end{array}$ & $\begin{array}{c}0.9291 \\
\text { (lag:) }\end{array}$ & $\begin{array}{c}0.9186 \\
\text { (lag:) }\end{array}$ & $\begin{array}{c}0.8949 \\
\text { (lag:) }\end{array}$ \\
\hline
\end{tabular}

Table 5. Result by using $800 \mathrm{~kW}$ synchronous condenser

\begin{tabular}{|l|l|l|l|l|l|l|}
\hline \multirow{2}{*}{\begin{tabular}{l}
\multirow{2}{*}{$\begin{array}{l}\text { of } \\
\text { load }\end{array}$} \\
\cline { 2 - 7 }
\end{tabular}} & $\begin{array}{l}\mathbf{0 . 7 5} \\
\text { (lead:) }\end{array}$ & $\begin{array}{l}\mathbf{0 . 8} \\
\text { (lead:) }\end{array}$ & $\begin{array}{l}\mathbf{0 . 8 5} \\
\text { (lead:) }\end{array}$ & $\begin{array}{l}\mathbf{0 . 9} \\
\text { (lead:) }\end{array}$ & $\begin{array}{l}\mathbf{0 . 9 5} \\
\text { (lead:) }\end{array}$ & $\mathbf{1}$ \\
\hline 50 & $\begin{array}{l}0.9972 \\
\text { (lead:) }\end{array}$ & $\begin{array}{l}0.9999 \\
\text { (lead:) }\end{array}$ & $\begin{array}{l}0.9989 \\
\text { (lag:) }\end{array}$ & $\begin{array}{l}0.9940 \\
\text { (lag:) }\end{array}$ & $\begin{array}{l}0.9837 \\
\text { (lag:) }\end{array}$ & $\begin{array}{l}0.9480 \\
\text { (lag:) }\end{array}$ \\
\hline \multirow{2}{*}{75} & $\begin{array}{l}0.9973 \\
\text { (lag:) }\end{array}$ & $\begin{array}{l}0.9927 \\
\text { (lag:) }\end{array}$ & $\begin{array}{l}0.9859 \\
\text { (lag:) }\end{array}$ & $\begin{array}{l}0.9768 \\
\text { (lag:) }\end{array}$ & $\begin{array}{l}0.9638 \\
\text { (lag:) }\end{array}$ & $\begin{array}{l}0.9292 \\
\text { (lag:) }\end{array}$ \\
\hline \multirow{2}{*}{100} & $\begin{array}{l}0.9859 \\
\text { (lag:) }\end{array}$ & $\begin{array}{l}0.9787 \\
\text { (lag:) }\end{array}$ & $\begin{array}{l}0.9703 \\
\text { (lag:) }\end{array}$ & $\begin{array}{l}0.9603 \\
\text { (lag:) }\end{array}$ & $\begin{array}{l}0.9474 \\
\text { (lag:) }\end{array}$ & $\begin{array}{l}0.9160 \\
\text { (lag:) }\end{array}$ \\
\hline \multirow{2}{*}{125} & $\begin{array}{l}0.9729 \\
\text { (lag:) }\end{array}$ & $\begin{array}{l}0.9649 \\
\text { (lag:) }\end{array}$ & $\begin{array}{l}0.9563 \\
\text { (lag:) }\end{array}$ & $\begin{array}{l}0.9465 \\
\text { (lag:) }\end{array}$ & $\begin{array}{l}0.9344 \\
\text { (lag:) }\end{array}$ & $\begin{array}{l}0.9063 \\
\text { (lag:) }\end{array}$ \\
\hline
\end{tabular}

Table 6. Result by using $1400 \mathrm{~kW}$ synchronous condenser

\begin{tabular}{|l|l|l|l|l|l|l|}
\hline \multirow{2}{*}{$\begin{array}{l}\text { \% } \\
\text { of } \\
\text { load }\end{array}$} & $\begin{array}{l}\mathbf{0 . 7 5} \\
\text { (lead:) }\end{array}$ & $\begin{array}{l}\mathbf{0 . 8} \\
\text { (lead:) }\end{array}$ & $\begin{array}{l}\mathbf{0 . 8 5} \\
\text { (lead:) }\end{array}$ & $\begin{array}{l}\mathbf{0 . 9} \\
\text { (lead:) }\end{array}$ & $\begin{array}{l}\mathbf{0 . 9 5} \\
\text { (lead:) }\end{array}$ & $\mathbf{1}$ \\
\hline \multirow{2}{*}{50} & $\begin{array}{l}0.9619 \\
\text { (lead:) }\end{array}$ & $\begin{array}{l}0.9797 \\
\text { (lead:) }\end{array}$ & $\begin{array}{l}0.9922 \\
\text { (lead:) }\end{array}$ & $\begin{array}{l}0.9990 \\
\text { (lead:) }\end{array}$ & $\begin{array}{l}0.9987 \\
\text { (lag:) }\end{array}$ & $\begin{array}{l}0.9704 \\
\text { (lag:) }\end{array}$ \\
\hline \multirow{2}{*}{75} & $\begin{array}{l}0.9912 \\
\text { (lead:) }\end{array}$ & $\begin{array}{l}0.9978 \\
\text { (lead:) }\end{array}$ & $\begin{array}{l}0.9999 \\
\text { (lead:) }\end{array}$ & $\begin{array}{l}0.9978 \\
\text { (lag:) }\end{array}$ & $\begin{array}{l}0.9896 \\
\text { (lag:) }\end{array}$ & $\begin{array}{l}0.9547 \\
\text { (lag:) }\end{array}$ \\
\hline \multirow{2}{*}{100} & $\begin{array}{l}0.9997 \\
\text { (lead:) }\end{array}$ & $\begin{array}{l}0.9995 \\
\text { (lag:) }\end{array}$ & $\begin{array}{l}0.9962 \\
\text { (lag:) }\end{array}$ & $\begin{array}{l}0.9894 \\
\text { (lag:) }\end{array}$ & $\begin{array}{l}0.9778 \\
\text { (lag:) }\end{array}$ & $\begin{array}{l}0.9419 \\
\text { (lag:) }\end{array}$ \\
\hline \multirow{2}{*}{125} & $\begin{array}{l}0.9984 \\
\text { (lag:) }\end{array}$ & $\begin{array}{l}0.9944 \\
\text { (lag:) }\end{array}$ & $\begin{array}{l}0.9882 \\
\text { (lag:) }\end{array}$ & $\begin{array}{l}0.9793 \\
\text { (lag:) }\end{array}$ & $\begin{array}{l}0.9665 \\
\text { (lag:) }\end{array}$ & $\begin{array}{l}0.9315 \\
\text { (lag:) }\end{array}$ \\
\hline
\end{tabular}

Table 7. Results by using $2400 \mathrm{~kW}$ synchronous condenser

\begin{tabular}{|l|l|l|l|l|l|l|}
\hline \multirow{2}{*}{$\begin{array}{l}\text { \% } \\
\text { of } \\
\text { load }\end{array}$} & $\begin{array}{l}\mathbf{0 . 7 5} \\
\text { (lead:) }\end{array}$ & $\begin{array}{l}\mathbf{0 . 8} \\
\text { (lead:) }\end{array}$ & $\begin{array}{l}\mathbf{0 . 8 5} \\
\text { (lead:) }\end{array}$ & $\begin{array}{l}\mathbf{0 . 9} \\
\text { (lead:) }\end{array}$ & $\begin{array}{l}\mathbf{0 . 9 5} \\
\text { (lead:) }\end{array}$ & $\mathbf{1}$ \\
\hline \multirow{2}{*}{50} & 0.9069 & 0.9381 & 0.9643 & 0.9847 & 0.9980 & 0.9852 \\
& (lead:) & (lead:) & (lead:) & (lead:) & $\begin{array}{l}\text { (lead:) } \\
\text { (lag:) }\end{array}$ \\
\hline 75 & 0.9491 & 0.9706 & 0.9867 & 0.9969 & 0.9998 & 0.9748 \\
& (lead:) & (lead:) & (lead:) & (lead:) & (lag:) & (lag:) \\
\hline \multirow{2}{*}{100} & 0.9750 & 0.9885 & 0.9969 & 0.9999 & $\begin{array}{l}0.9963 \\
\text { (lag:) }\end{array}$ & $\begin{array}{l}0.9649 \\
\text { (lag:) }\end{array}$ \\
\hline \multirow{2}{*}{125} & $\begin{array}{l}\text { (lead:) } \\
\text { (lead:) }\end{array}$ & $\begin{array}{llll}\text { (lead:) } \\
\text { (lead:) }\end{array}$ & $\begin{array}{l}\text { (lead:) } \\
\text { (lead:) }\end{array}$ & $\begin{array}{l}\text { (lead:) } \\
\text { (lag:) }\end{array}$ & $\begin{array}{l}0.9905 \\
\text { (lag:) }\end{array}$ & $\begin{array}{l}0.9559 \\
\text { (lag:) }\end{array}$ \\
\hline
\end{tabular}

The power factor using the ratings of $800 \mathrm{~kW}, 1400 \mathrm{~kW}$ and $2400 \mathrm{~kW}$ are within limit, but they are large and more cost. The range of power factor by using $600 \mathrm{~kW}$ synchronous motor can operate the plant load changing within restricted region. There are of four-load shedding at the plant. If it is required to shed the load, varying the power factor of the synchronous motor can be affective the plant operation.
Results for load shedding by using $600 \mathrm{~kW}$ synchronous motor are shown in Table 9 Instead of $600 \mathrm{~kW}$ synchronous motor,

Table 8. Results for percent load changing by using $600 \mathrm{~kW}$ synchronous condenser

\begin{tabular}{|c|c|}
\hline \% change of load & $\begin{array}{c}\text { p.f of synchronous condenser } \\
\text { (lead:) }\end{array}$ \\
\hline 50 & $0.95 \sim 0.75$ \\
\hline 75 & $0.9 \sim 0.75$ \\
\hline 100 & $0.85 \sim 0.75$ \\
\hline 125 & 0.75 \\
\hline
\end{tabular}

Table 9. Results for load shedding by using $600 \mathrm{~kW}$ synchronous condenser

\begin{tabular}{|c|c|c|}
\hline \multicolumn{2}{|c|}{ Induction motors in used } & \multirow{2}{*}{$\begin{array}{c}\text { p.f of synchronous } \\
\text { condenser (Lead:) }\end{array}$} \\
\cline { 1 - 2 } kW & No: & 0.99 \\
\hline 110 & 3 & 0.989 \\
\hline 110 & 3 & \\
250 & 3 & 0.835 \\
\hline 110 & 3 & \\
250 & 3 & \\
200 & 3 & 0.75 \\
\hline 110 & 3 & \\
250 & 3 & \\
200 & 3 & \\
160 & 1 & \\
\hline
\end{tabular}

Table 10. Results for load shedding by using three numbers of $200 \mathrm{~kW}$ synchronous condensers

\begin{tabular}{|c|c|c|}
\hline \multicolumn{2}{|c|}{ Induction motors in used } & \multirow{2}{*}{$\begin{array}{c}\text { No: of synchronous } \\
\text { condenser }\end{array}$} \\
\hline $\mathbf{k W}$ & No: & \\
\hline 110 & 3 & \multirow{5}{*}{1} \\
\hline 110 & 3 & \\
\hline 200 & 3 & \\
\hline 200 & 3 & \\
\hline 160 & 1 & \\
\hline 110 & 3 & \multirow{8}{*}{2} \\
\hline 250 & 3 & \\
\hline 250 & 3 & \\
\hline 200 & 3 & \\
\hline 110 & 3 & \\
\hline 250 & 3 & \\
\hline 200 & 3 & \\
\hline 160 & 1 & \\
\hline
\end{tabular}

three numbers of $200 \mathrm{~kW}$ synchronous motor can be used in the plant. And the results are shown in Table 10. In this table, only one $200 \mathrm{~kW}$ synchronous motor can be used for running with three numbers of $110 \mathrm{~kW}$ induction motors or three numbers of $110 \mathrm{~kW}$ and three numbers of $200 \mathrm{~kW}$ induction motors or three number of $200 \mathrm{~kW}$ and a $160 \mathrm{~kW}$ induction motors. Other load conditions are also shown in the table. 


\section{DISCUSSION}

Loads used in domestics or industries are mostly inductive loads. Induction motor is that load. As inductive effect, power factor is lagging and it causes the system energy losses. Due to the effect, the load current is high, unload balancing is occurred and power quality is bad. From the point of view of economic, it also costs for the energy losses. For all cases, power factor improvement is needed for the system and compensators are essentially required.

In the study, a synchronous condenser or synchronous motor is used in parallel with induction motors as a compensator in the plant. As there are all type of induction motors, the plant overall power factor is also lagging. So synchronous motor with power factor leading is used to improve the plant power factor. Although unity power factor is the best one for the system, it is difficult to become nearly unity power factor of the system in practice but in theory. So there is limited range of plant power factor between unity and 0.95 lagging. For that why, the study is to drive the plant to be with limited power factor range.

To compensate the lagging power factor load to be unity one, the synchronous condenser must operate in leading power factor mode. Higher lagging power factor load needs higher leading power factor compensator. To be high power factor leading synchronous condenser needs larger excitation. So the study has not tried to be the plant power factor to be unity, but to be within the limit nearly 0.97 and 0.96 .

In the study, there is only one synchronous condenser of $600 \mathrm{~kW}$ in use to compensate the plant. And it also shows the data for the plant load changing. According to the results data, by varying the power factor of synchronous condenser, the plant power factor will be its limited range when it is needed to reduce or increase its load. As the limited value is within the range, six ratings of synchronous condenser power factor can drive the plant load in changed to be acceptable value.

\section{CONCLUSION}

In comparison with other compensators such as capacitor banks, reactors, synchronous condenser has some advantages in varying power factor. When load changing is encountered in the system, by easily varying the excitation system of synchronous condensers, the desired value has been in the system. Others compensators need to change add or reduce the components. But synchronous condenser is rotating machine, it can be needed some maintenance and it will cause some losses. As the operation of synchronous condenser can vary, it needs operator. So sometime fault operations may encounter in the system.

Instead of varying one synchronous condenser, a number of synchronous condensers being in used together should be studied in further study. The comparison should be made in the cost of capacitor banks and the cost of synchronous condensers, and the unit changes for using synchronous condensers. As the initial cost of synchronous condenser is large and as well its operating cost, it is needed to know about the synchronous machine, how to operate the machine, and how to control the machine. And as power factor adjustment is done by exciter, excitation systems of the machine are also important facts. As instead of $600 \mathrm{~kW}$ synchronous condenser, three numbers of $200 \mathrm{~kW}$ synchronous condenser can be used, further study should be done to find the merits using low rating of synchronous condenser in numbers.

\section{ACKNOWLEDGMENTS}

The author would like to express thanks to her parents for their noble support and encouragement. Next, the author would like to gratitude to the teachers who taught her everything. Then, the author would also like to express gratitude to relatives and friends who helped her along her life.

\section{REFERENCES}

[1] C. Sankaran, Power Quality, CRC Press LLC, Printed in the United States of America, 2002.

[2] +5V-Powered, Multichannel RS-232 Drivers/Receivers, May 2005, http://www.maxim.ic.com/packages..

[3] Data Sheet of Silicon Epitaxial Planner Type Diode, 1S1585_1S1588, February 2006, http://www.datasheetarchive.com.

[4] John Webb/Kevin Greshock, Industrial Control Electronic, May 2003.

[5] Leonard L. Grigsby, Electric Power Generation, Transmission and Distribution, Talor and Francis Group, CRC Press LLC, 2006.

[6] R. Sastry Vedam Mulukutla and S. Sarma, Power Quality VAR Compensation in Power Systems, Tallor and Francis Group, CRC Press LLC, 2009.

[7] Roger C.Dugan, Mark F. McGranaghan, Surya Santoso, H. Wayna Beaty, Electrical Power System Quality, Second edition, 2004 by Mc Graw-Hill

[8] Hand Book of Power Quality, edited by Angelo Baggini, University of Bergamo, Italy, copyright by John Wiley and Sons Ltd, 2008. 